\title{
Standard test procedure to characterise different filter media under energy consideration
}

\author{
W. Hoeflinger \& T. Laminger \\ Institute of Chemical Engineering, Vienna University of Technology, \\ Austria
}

\begin{abstract}
In the context of increasing energy prices and the imperative of reducing $\mathrm{CO}_{2}$ emissions, the energy consumption also related to dust separators has become the focus of attention. Further, new stricter regulations for the particle concentration in the environmental air (PM10 PM2.5) require the improvement of existing dust separators. The improvement of the separation efficiency of dust separators is always associated with a higher energy input, in case of a filtering device with an increase of the pressure drop and other energy inputs during the filtration procedure. Especially for air depth-filters, used for applications with low dust concentrations (e.g. ventilation of living and working spaces), a new guideline "EUROVENT 4/11" [1] for energy efficient operation has already been developed. With this method a classification of different filter media concerning their energy consumption and their separation efficiency in relation to each other can be done.

For applications with high dust concentrations (e.g. waste gas cleaning of combustion or cement plants), where so called cleanable dust filter media are in use, a classification of different cleanable dust filter media can be done by the standards ISO11057:2011 [2] and VDI 3926 [3]. But these standards make no statement to their relative energy consumption. In this work an approach was made to classify different filter media concerning their relative energy consumption in connection with their separation efficiency.

With a special test rig, which are also used in ISO11057:2011 [2], a total energy consumption value $\left[\mathrm{Wh} / \mathrm{m}^{3}\right]$ related to the air volume throughput and the corresponding separation efficiency was evaluated over 30 filtration cycles. This energy value comprises all relevant energy consumption parts (fan energy and
\end{abstract}


cleaning energy) by a single value. Determining this value and also the associated separation efficiency for each filter medium, different filter media can be classified concerning their quality.

Keywords: energy consumption, cleanable dust filter, clean gas.

\section{Introduction}

In the context of increasing energy prices and the imperative of reducing $\mathrm{CO}_{2}$ emissions, the energy consumption related to separators has become the focus of attention. Optimization of filter apparatus for minimum energy consumption is becoming more and more an issue for filter design. To remove dust from waste gases, especially for high dust concentrations (e.g. waste gas cleaning of combustion or cement plants), so called cleanable dust filter media are in use. For such filter systems an improvement of the filtration efficiency goes hand in hand with increasing energy consumption. To classify different filter media knowledge of their relative energy consumption in connection with the filtration efficiency is needed.

Especially for air filters, used for applications of ventilation of living and working spaces (low dust concentrations), a new guideline "EUROVENT 4/11" [1] has been developed, by which different filter media can be classified concerning their energy-efficient operation. So far, air filters have been only classified by their average particle collection efficiency using special laboratory equipment according to the European standard DIN EN 779 [4].

Following this new EUROVENT 4/11 guideline, the yearly energy consumption of air filters (W) can be determined as a function of the volume flow rate $\left(\mathrm{q}_{\mathrm{v}}\right)$, the fan efficiency $(\eta)$, the operation time $(\mathrm{t})$ and the average pressure drop $(\overline{\Delta p})$. The related energy consumption during a certain period of time (e.g. a year) can be calculated from the integral average of the pressure drop according to equation (1). The integral average of the pressure drop is needed as, due to the dust loading during operation, the pressure drop of an air-filter is increasing. Thereby, the average pressure drop results by a laboratory filter test according to DIN EN 779.

$$
W=\frac{q_{V} \cdot \overline{\Delta p} \cdot t}{\eta \cdot 1000}
$$

To classify the filter media, the calculated yearly energy consumption (equation (1)) shall be compared with a grid of defined maximum energy consumption values. Thereby, the limits of each energy efficiency class depend on the achieved filter media class with a defined separation efficiency, which was reached after a DIN EN 779 classification (G1 to F9) [1].

Such an evaluation procedure, focusing on separation efficiency and energy consumption, is also desirable for classification of cleanable filter media. The test equipments and the test methods from the ISO11057:2011 [2] or VDI 3926:2009 [3] can be used as suitable testers. These guidelines describe a filter test rig and a test procedure to determine the behaviour of the residual pressure 
drop and the filtration cycle duration over the number of the filtration cycles as well as the mean clean gas dust concentration.

However, no workable information on the energy consumption of such a filter test can be obtained, which shall be the content of the next section.

\section{Development of an energy consumption value for evaluation from ISO11057:2011 or VDI3926 test runs}

To compare different filter media in a relative way with regard to the energy consumption, the total energy needed has to be related to the air volume throughput $\left[\mathrm{Wh} / \mathrm{m}^{3}\right]$. Such a total energy consumption value, related to air volume throughput $\left[\mathrm{W} / \mathrm{hm}^{3}\right]$ should comprise all relevant energy relevant consumptions (fan energy and jet-pulse energy) achieved from the test run by a single value. Having determined this value and also the associated average particle throughput or the particle separation for different filter media, a classification can be done concerning their quality.

The test is running over 30 filtration cycles, where during the filtration time interval the pressure drop increases until a certain maximum pressure drop is reached. Thereafter the filter medium is cleaned-off by back pulse and the next cycle starts. The test equipment in accordance with the ISO11057:2011 and VDI 3926 guide-lines are shown in Figure 1. As test dust is used Sasol Pural NF (Aluminia-oxid), which is prescribed also in the guidelines with a median mass particle size $\left(\mathrm{d}_{50,3}\right)$ of 4.5 microns. According to the guidelines, for a standard classification of different filter media, all filter media must be tested with the same special test dust. This test dust together with the disperser ensures also constant and reproducible particle size distributions and concentrations over test time.

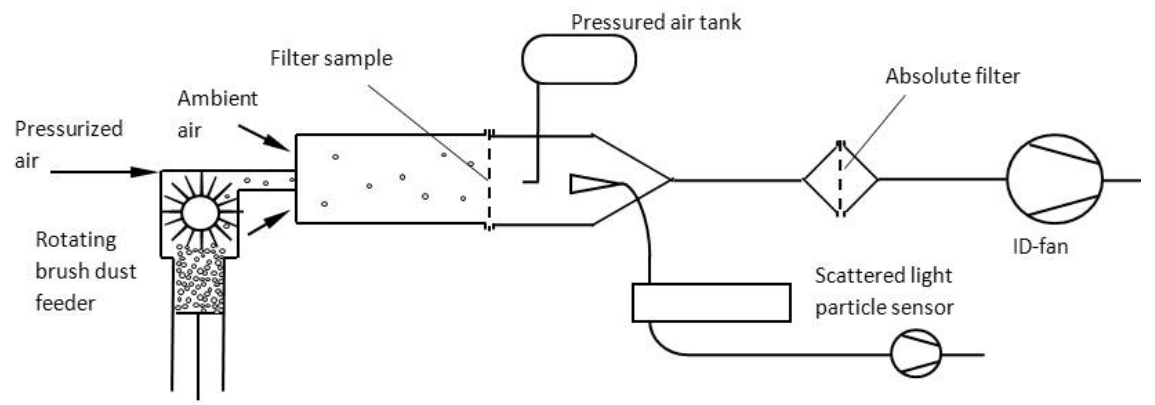

Figure 1: Filter test equipment to evaluate the relative total energy consumption and the relative particle penetration behaviour of different filter media.

From a filter test run, the total energy consumption $\left(\mathrm{E}_{\mathrm{tot}, \mathrm{n}}\right)$ [Wh] during the total test duration $\left(\mathrm{t}_{\mathrm{tot}, \mathrm{n}}\right)[\mathrm{h}]$, from beginning of the filter test to the end of the 
filtration cycle $n$, can be defined by the averaged total power consumption $\left(\mathrm{P}_{\text {tot,nn }}\right)$ over all filtration cycles $n$ multiplied by the total test duration $\left(t_{\text {tot, }}\right)$ :

$$
E_{t o t, n}=P_{t o t, n} \cdot t_{t o t, n}
$$

The total test duration $\left(t_{t o t, n}\right)$ can be calculated by adding all filtration cycle durations $\left(\mathrm{t}_{\text {cycle, }, \mathrm{i}}\right)[\mathrm{h}](\mathrm{i}=1$ to $\mathrm{n})$ :

$$
t_{t o t, n}=\sum_{i=1}^{n} t_{c y c l e, i}
$$

The power consumption averaged from the beginning of a filter test to the end of the filtration cycle $n\left(\mathrm{P}_{\mathrm{tot}, \mathrm{n}}\right)$ [W] can be calculated by summing up the power consumption of the ID-fan $\left(\mathrm{P}_{\mathrm{fan}, \mathrm{n}}\right)[\mathrm{W}]$ and the power consumption needed for the compressed air of the jet-pulse cleaning $\left(\mathrm{P}_{\text {cleaning, } n}\right)[\mathrm{W}]$ :

$$
P_{\text {tot }, n}=P_{\text {fan }, n}+P_{\text {cleaning }, n}
$$

The power consumption of the fan averaged from the beginning of a filter test to the end of the filtration cycle $n\left(\mathrm{P}_{\text {fan, }}\right)$ depends mainly on the pressure drop across the tested filter medium averaged from the beginning of a filter test to the end of the filtration cycle $\mathrm{n}\left(\Delta \bar{p}_{\text {filter }, n}\right)[\mathrm{Pa}]$ multiplied by the air volume flow $(\dot{V})\left[\mathrm{m}^{3} / \mathrm{h}\right]$ :

$$
P_{\text {fan. } n}=\Delta \bar{p}_{\text {filter }, n} \cdot \dot{V}
$$

Considering the changes of the filter pressure drop during the filter test, for the calculation of the averaged power consumption of the ventilator an averaged pressure drop $\left(\Delta \bar{p}_{\text {filter, },}\right)$ can be used. The averaged pressure drop from the beginning of a filter test to the end of the filtration cycle $\mathrm{n}\left(\Delta \bar{p}_{f i l t e r, n}\right)$ can be calculated by a numeric integration of the time-discrete measured pressure drop across the tested filter medium $\left(\mathrm{p}_{\text {filter, }}\right)[\mathrm{Pa}]$. Thereby, the measurement time interval $\left(\mathrm{MTI}_{\mathrm{p}}\right)[\mathrm{s}]$ is defined by the used pressure drop measurement device (e.g. $\mathrm{MTI}_{\mathrm{p}}=3 \mathrm{sec}$ ) (Figure 2):

$$
\Delta \bar{p}_{\text {filter }, n}=\frac{1}{t_{\text {tot }, n} / M^{\prime} I_{p}} \cdot \sum_{i=1}^{t_{t o t, n} / M T I_{p}} p_{\text {filter }, i}
$$

To calculate the averaged power consumption for the pressurized air of the jet-pulse cleaning $\left(\mathrm{P}_{\text {cleaning,n }}\right)[\mathrm{W}]$ of the filter test from the beginning of a filter test to the end of the filtration cycle $\mathrm{n}$, the following assumptions are made: For each filtration cycle a certain power consumption is needed to provide pressurized air of the jet-pulse cleaning of the filter medium. This power consumption, which depends on the pressurized air tank volume $\left(\mathrm{V}_{\text {tank }}\right)\left[\mathrm{m}^{3}\right]$ and on the pressure difference (before and after the jet-pulse) of the pressurized air within the air tank $\left(\Delta \mathrm{p}_{\text {tank }}\right)[\mathrm{Pa}]$, can be related to the filtration cycle duration. 


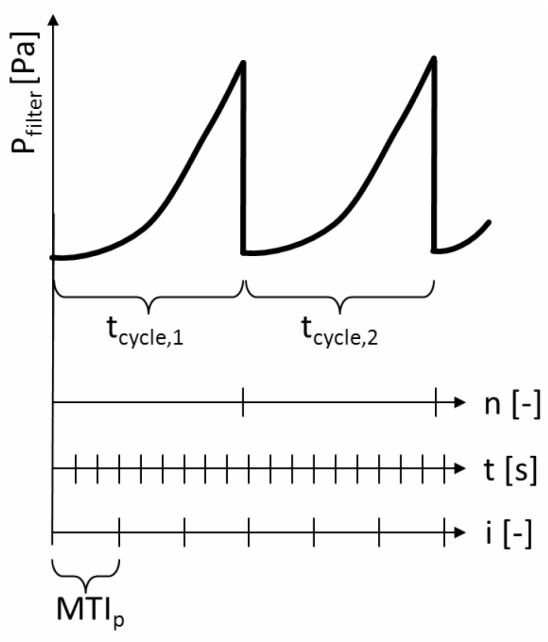

Figure 2: Development of the pressure drop across the filter medium $\left(\mathrm{p}_{\text {filter }}\right)$ over the time $(\mathrm{t})$, the filtration cycle number (n) and the measurement time intervals (i).

It is assumed that the pressurized air tank volume $\mathrm{V}_{\text {tank }}$ and the tank pressure difference $\Delta \mathrm{p}_{\text {tank }}$ are constant for each filtration cycle. Therefore, for a number of $\mathrm{n}$ filtration cycles, the averaged power consumption for the compressed air of the jet-pulse cleaning $\left(\mathrm{P}_{\text {cleaning,n }}\right)$ can be calculated as follows:

$$
P_{\text {cleaning }, n}=\frac{\Delta p_{\text {tank }} \cdot V_{\text {tank }} \cdot n}{\sum_{i=1}^{n} t_{c y c l e, i}}
$$

The calculation of the power consumption from the first up to a certain filtration cycle number $\mathrm{n}\left(\mathrm{P}_{\text {tot, }, \mathrm{n}}\right)$ (equation $\left.(4)\right)$ can now be given in more detail:

$$
P_{\text {tot }, n}=\Delta \bar{p}_{\text {filter }, n} \cdot \dot{V}+\frac{\Delta p_{\text {tank }} \cdot V_{\text {tank }} \cdot n}{\sum_{i=1}^{n} t_{\text {cycle }, i}}
$$

The total energy consumption $\left(\mathrm{E}_{\mathrm{tot}, \mathrm{n}}\right)$ can be related to the total air volume throughput during the test $\left(\mathrm{V}_{\text {tot,n }}\right)\left[\mathrm{m}^{3}\right]$ to get the volume-related total energy consumption value $\left(\mathrm{E}_{\mathrm{V}, \mathrm{tot}, \mathrm{n}}\right)\left[\mathrm{Wh} \mathrm{m}^{-3}\right]$ :

$$
E_{V, t o t, n}=\frac{E_{t o t, n}}{V_{t o t, n}}
$$

With a constant air volume flow $(\dot{V})$, the total air volume throughput can be expressed by multiplying the air volume flow with the total duration $\left(\mathrm{t}_{\text {tot,n }}\right)$, which gives for the volume-related total energy consumption value: 


$$
E_{V, t o t, n}=\frac{E_{t o t, n}}{V_{t o t, n}}=\frac{E_{t o t, n}}{\dot{V} \cdot t_{t o t, n}}=\frac{P_{t o t, n} \cdot t_{t o t, n}}{\dot{V} \cdot t_{t o t, n}}=\frac{P_{t o t, n}}{\dot{V}}
$$

Now, including equation (8) in equation (10), the volume-related total energy consumption from the beginning of a filter test to the end of the filtration cycle $n$ is formulated as follows:

$$
E_{V, t o t, n}=\Delta \bar{p}_{\text {filter }, n}+\frac{\Delta p_{\text {tank } k} \cdot V_{\text {tank }} \cdot n}{\dot{V} \cdot \sum_{i=1}^{n} t_{c y c l e, i}}
$$

The volume-related total energy consumption value $\mathrm{E}_{\mathrm{V}, \mathrm{tot}, \mathrm{n}}$ includes in one single value the evolution of the pressure drop of the filter medium and the cleaning energy resulted from the pressurized air consumption for a considered total filtration number $\mathrm{n}$.

This can be used, for example, to compare different filter media for the first 30 filtration cycles of an ISO11057:2011 filter test or for the 30 filtration cycles following a filter aging procedure. Thereby, $\mathrm{E}_{\mathrm{V}, \mathrm{tot}, 30}$ can be calculated as follows:

$$
E_{V, \text { tot }, 30}=\Delta \bar{p}_{\text {filter }, 30}+\frac{\Delta p_{\text {tank }} \cdot V_{\text {tank }} \cdot 30}{\dot{V} \cdot \sum_{i=1}^{30} t_{\text {cycle }, i}}
$$

As it can be seen in equation (12), a relative high averaged pressure drop of a filter medium within the first 30 filtration cycles (due to e.g. patchy cleaning) will increase the volume-related total energy consumption value. Whereas, relative longer filtration cycle durations $t_{c y c l e, i}$ (e.g. predominant depth filtration behaviour) will decrease $\mathrm{E}_{\mathrm{V}, \mathrm{tot}, \mathrm{n}}$.

In order to compare different filter media, the evaluation of $\mathrm{E}_{\mathrm{V}, \text { tot, }}$ can also be done for the 30 filtration cycles following a filter ageing test procedure (e.g. 2.500 jet-pulse cleanings at an interval of $20 \mathrm{~s}$ each).

\section{Experimental determination of the volume-related total energy consumption and the filtration efficiency}

In order to compare different filter media concerning their total energy consumption in connection with their mean clean gas concentration (used as main indicator for the filtration behaviour of the filter media), filter tests on the test rig were performed. The test parameters, which correspond to ISO11057:2011, are given in Table 1. Thereby, the used test dust (Pural NF) is a very fine alumina monohydrate powder in the range between 1 and 20 micron with a mass mean diameter $\left(\mathrm{d}_{50,3}\right)$ of $4.5 \mu \mathrm{m}$.

The test procedure for each filter test was realized as follows: the dust loaded raw gas stream was generated by drawing ambient air into a horizontal raw gas channel, where test dust with exactly adjusted amounts was added by using a rotating brush dust feeder. The dust-loaded raw gas stream passed through the filter sample. The circular filter sample which has to be investigated was 
Table 1: Test parameters of the filter tests (in accordance with ISO11057:2001).

\begin{tabular}{|l|c|c|}
\hline Filter face velocity & $\mathrm{m}^{3} /\left(\mathrm{m}^{2} \mathrm{~h}\right)$ & 120 \\
\hline Dust concentration (raw gas) & $\mathrm{g} / \mathrm{m}^{3}$ & 5 \\
\hline Test dust & \multicolumn{2}{|c|}{$\begin{array}{r}\text { Sasol Pural NF } \\
\mathrm{d}_{10,3}=1.2 \mu \mathrm{m}, \mathrm{d}_{50,3}=4.5 \mu \mathrm{m}, \\
\mathrm{d}_{90,3}=21.0 \mu \mathrm{m}\end{array}$} \\
\hline $\begin{array}{l}\text { Pressure drop prior to pulse-jet } \\
\text { cleaning }\end{array}$ & $\mathrm{Pa}$ & 1000 \\
\hline Tank pressure & $\mathrm{bar}$ & 5 \\
\hline Tank volume $\left(\mathrm{V}_{\text {tank }}\right)$ & 1 & 2 \\
\hline $\begin{array}{l}\text { Tank pressure difference before } \\
\text { and after the jet-pulse }\left(\Delta p_{\text {tank }}\right)\end{array}$ & $\mathrm{bar}$ & 0.26 \\
\hline Valve opening time & $\mathrm{ms}$ & 60 \\
\hline Number of filtration cycles & {$[-]$} & 30 \\
\hline Sample area (effective) & $\mathrm{m}^{2}$ & 0.0177 \\
\hline
\end{tabular}

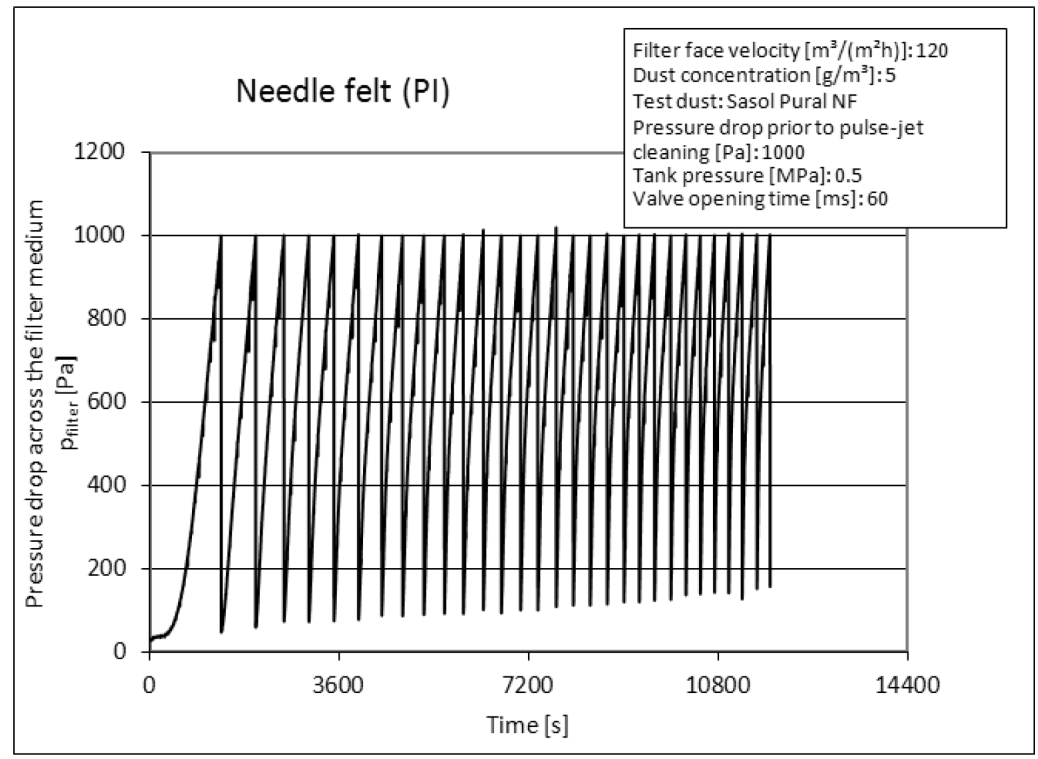

Figure 3: Time development of the pressure drop of the filter medium.

mounted flat in a filter fixture and during filtration the dust particles deposit as dust filter cake over the filter sample.

The pressure drop across the filter sample was measured every three seconds $(\mathrm{MTI}=3 \mathrm{sec})-$ see Figure 3 . When a pre-determined maximum pressure drop across the dust loaded filter sample was reached the filter cake was removed by 
pulse-jet cleaning, using a pulse of compressed air coming from the clean gas side and the pressure drop felt down to a low residual pressure drop. After cleaning the filter medium, the next filtration cycle started and the pressure drop increased again, until the total number of a pre-set filtration cycles had been reached (e.g. 30 filtration cycles).

From this curve (Figure 3) the course of the residual pressure drop and filtration cycle duration can derived (Figure 4).

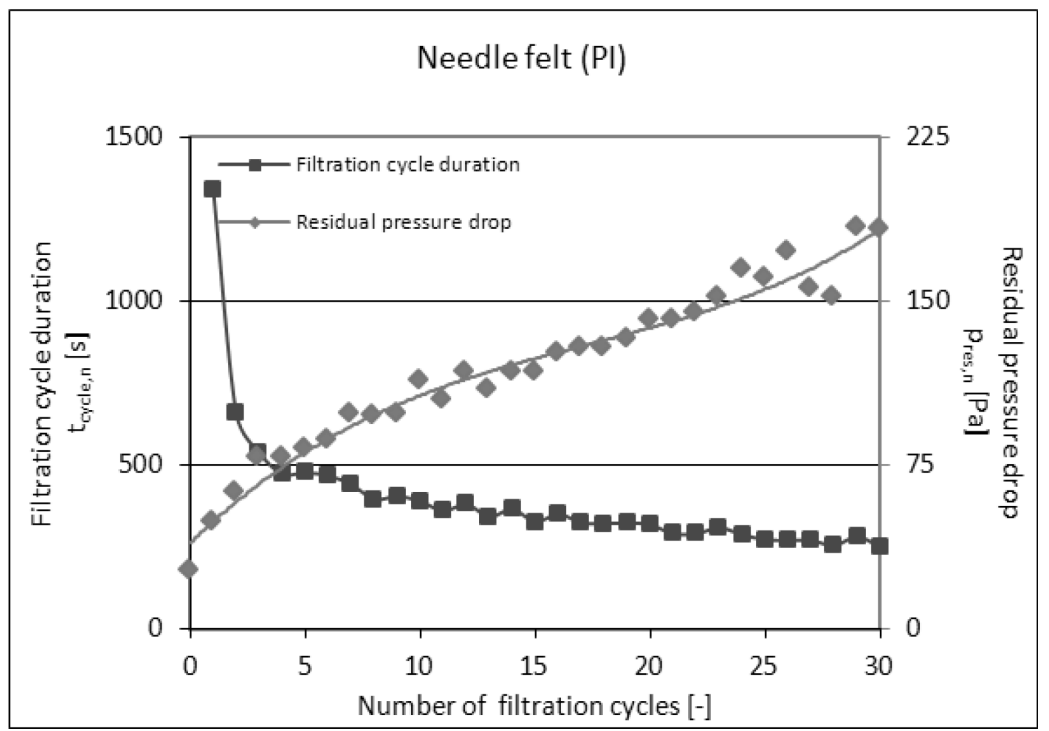

Figure 4: Residual pressure drop and filtration cycle duration over the number of filtration cycles.

In order to determine the particle size distribution of the clean gas, a sample stream was sucked off from the clean gas. The particle size distribution of the clean gas was measured every 60 seconds by a scattered light particle counter sizer (PCS) to determine the clean gas concentration $\left(\mathrm{c}_{\text {clean }}\right)$ over the test time (Figure 5$)$. The averaged mean clean gas concentration $\left(\mathrm{c}_{\text {clean,mean, }}\right)$ from the beginning of a filter test up to a certain filtration cycle number $n$ was calculated by a numeric integration of the time discretely measured clean gas mass concentrations $\left(\mathrm{c}_{\mathrm{clean}, \mathrm{i}}\right)$ for all filtration cycles $(\mathrm{n}=1$ to 30$)$ :

$$
c_{\text {clean,mean }, n}=\frac{1}{t_{\text {tot }, n} / M T I_{c, \text { clean }}} \cdot \sum_{i=1}^{t_{\text {tot }, n} / M T I_{c, \text { clean }}} c_{\text {clean }, i}
$$

Using equations (11) and (13), the development volume-related energy consumption value over the filtration cycle number could be calculated and was plotted together with the development of the mean clean gas concentration (Figure 6). 


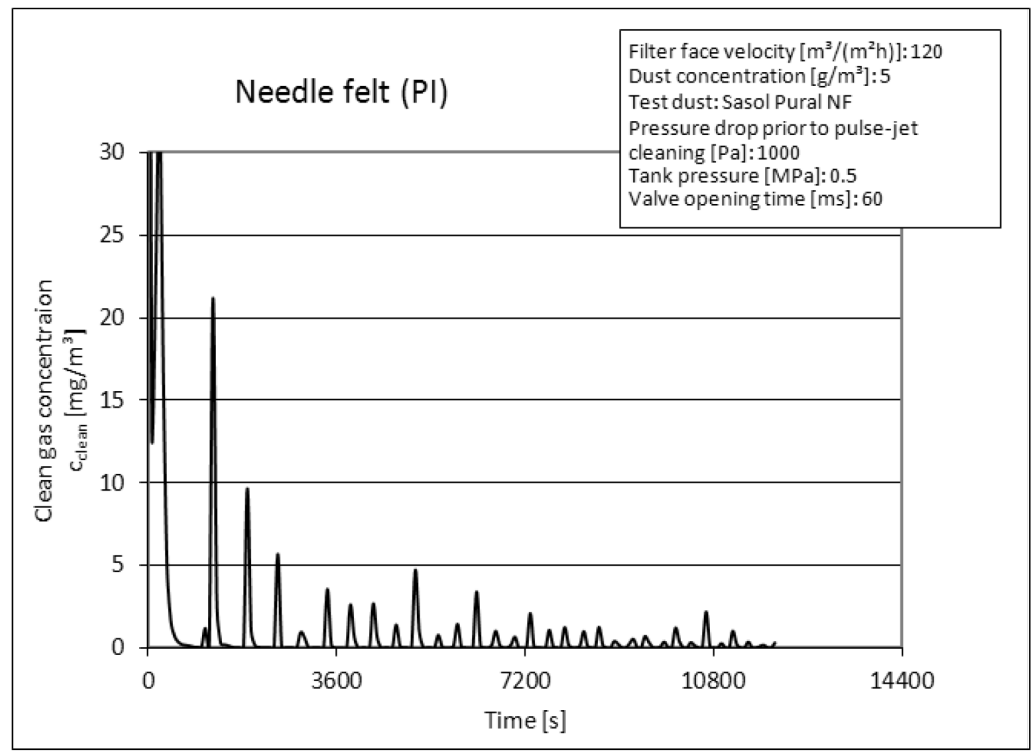

Figure 5: Clean gas concentration for differential pressure controlled filter cleaning.

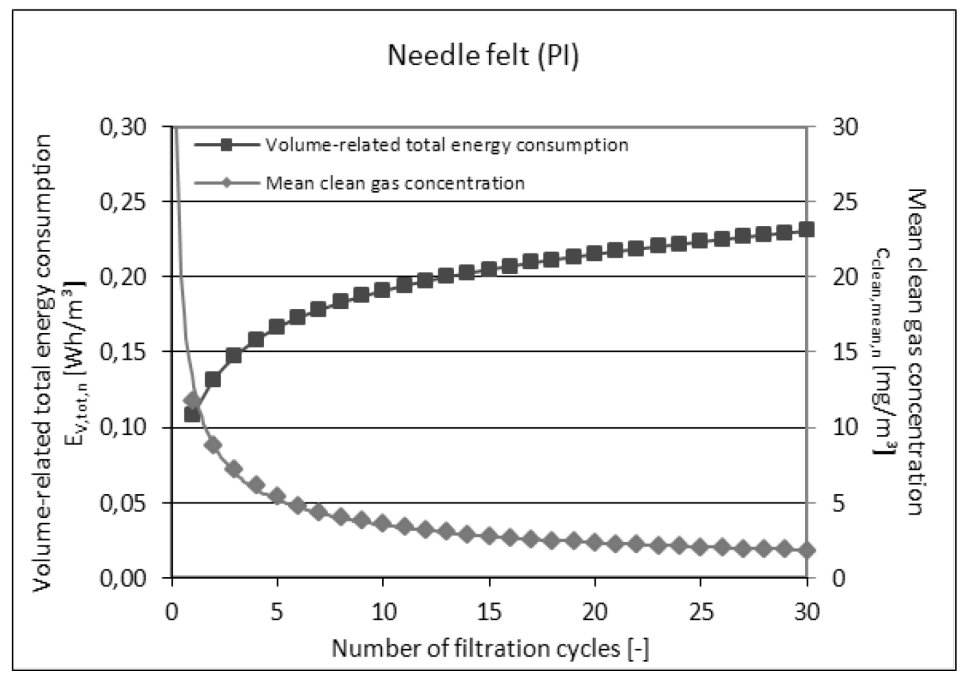

Figure 6: Volume related total energy consumption and mean clean gas concentration over the number of filtration. 


\section{Experimental results of the total energy consumption and mean clean gas concentration of different filter media}

In this test series ten different filter media (five different membranes and five different needle felts) were tested according to the test procedure (section 3). In Figure 7 these filter media are compared regarding their relative energy consumption value $\left(\mathrm{E}_{\mathrm{v}, \text { tot, } \mathrm{n}}\right)$ and their achieved mean clean gas concentration $\left(\mathrm{c}_{\text {clean,mean, }}\right)$. In Figure 7 can be seen that all ten filter media differ from each other: There are filter media which have a very low mean clean gas concentration (e.g. Membrane 1 and Membrane 2) and a few filter media with a volume-related energy consumption value below $0.25 \mathrm{Wh} / \mathrm{m}^{3}$ (e.g. Membrane 1 , Membrane 2, Needle felt 1 to 3).

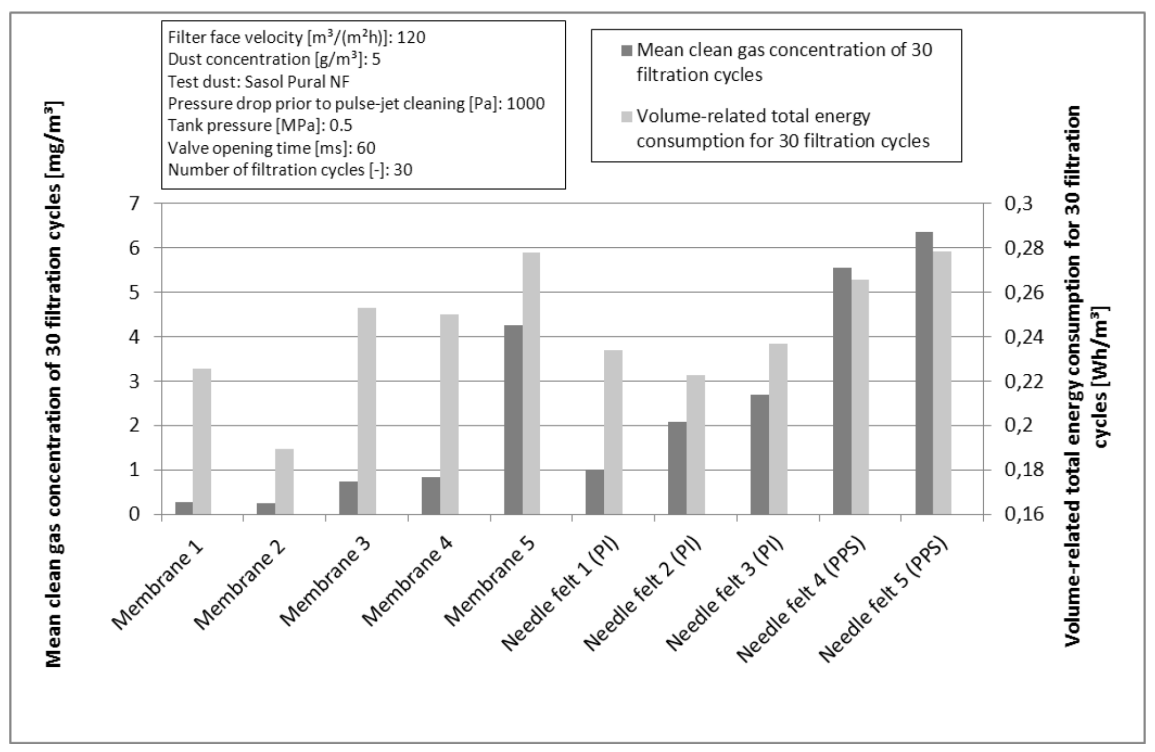

Figure 7: Comparison of the volume related total energy consumption and the mean clean gas concentration of the tested filter media (resulted after 30 filtration cycles).

Some filter media (Membrane 5, Needle felt 4 and Needle felt 5) protrude from the other: These three filter media have relative high mean clean gas concentration values and high volume-related total energy consumption values, which are of course the worst tested filter media in this test series.

The volume-related total energy consumption values can further be used to classify the most adequate filter medium for a certain limit of mean clean gas concentration. For a fictive limit value of $<1 \mathrm{mg} / \mathrm{m}^{3}$ mean clean gas concentration, the Membrane filter media 1 to 4 are the only filter media which fulfil this criterion. Concerning their energy consumption values $\mathrm{E}_{\mathrm{v}, \mathrm{tot}, \mathrm{n}}$, the best 
choice will be the Membrane 2 which has the lowest energy consumption value of the selected filter media.

\section{Summary}

In order to compare different cleanable dust filter media under standard laboratory conditions, the ISO 11057:2011 or the VDI 3926 describes a test rig and a test procedure to determine the development of the residual pressure drop the cycle durations and over the number of filtration cycles and the separation efficiency. The first two parameters are relevant concerning the energy consumption and could have different developments over time. Therefore, it is difficult to compare different filter media to conclude which one has the lowest energy consumption for specified separation efficiency.

Therefore, in this work within these standard test procedures a volume-related energy consumption value $\left(\mathrm{E}_{\mathrm{v}, \text { tot, } \mathrm{n}}\right)$ was developed to have only a single value to compare different cleanable dust filter media with regard to their energy consumption. This volume-related energy consumption value added the needed average energy for the fan and the average energy for the jet-pulse cleaning gained from the standard filter test run. Further the measured, averaged clean gas concentration $\left(\mathrm{c}_{\mathrm{clean}, \mathrm{mean}, \mathrm{n}}\right)$ was used to determine the particle separation behaviour of the filter medium.

Different filter media (membranes and needle felts) were tested (according to ISO 11057:2011). After 30 filtration cycles the parameter values of $E_{v, t o t, n}$ and $\mathrm{c}_{\text {clean,mean, } \mathrm{n}}$ of all tested filter media were listed. It was shown, that with these parameters a classification of different filter media with regard to the energy consumption and for a given clean gas concentration limit can be easily done. This procedure can be also executed for the 30 filtration cycles after the ageing procedure described in the ISO 11057:2011.

\section{References}

[1] EUROVENT 4/11, Energy efficiency classification of air filters for general ventilation purposes; First edition 2011.

[2] ISO 11057 (2011), Air quality - Test method for filtration characterization of cleanable filter media. Berlin, Germany: Beuth Verlag.

[3] VDI 3926 (2009), Testing of cleanable filter media - Standard test for the evaluation of cleanable filter media, Berlin, Germany: Beuth Verlag.

[4] DIN EN 779 (2009), Particulate air filters for general ventilation Determination of the filtration performance, Berlin, Germany: Beuth Verlag. 